\title{
The Barban-Vehov Theorem in Arithmetic Progressions
}

\author{
V. Kumar Murty
}

To the memory of S. Srinivasan

\begin{abstract}
A result of Barban-Vehov (and independently Motohashi) gives an estimate for the mean square of a sequence related to Selberg's sieve. This upper bound was refined to an asymptotic formula by S. Graham in 1978. In 1992, I made the observation that Graham's method can be used to obtain an asymptotic formula when the sum is restricted to an arithmetic progression. This formula immediately gives a version of the Brun-Titchmarsh theorem. I am taking the occasion of a volume in honour of my friend S. Srinivasan to revisit and publish this observation in the hope that it might still be of interest.
\end{abstract}

Keywords. Selberg's sieve, Brun-Titchmarsh theorem, arithmetic progressions

2010 Mathematics Subject Classification. Primary 11N37, 11N13 ; Secondary 11B25, 11N35, 11N69

\section{Introduction}

Let $1 \leq z_{1} \leq z_{2}$ and define for $i=1,2$,

$$
\Lambda_{i}(n)= \begin{cases}\mu(n) \log \frac{z_{i}}{n} & \text { if } n \leq z_{i} \\ 0 & \text { if } n>z_{i}\end{cases}
$$

Also, set

$$
\lambda_{n}=\frac{\Lambda_{2}(n)-\Lambda_{1}(n)}{\log z_{2} / z_{1}}
$$

and

$$
a(n)=\sum_{d \mid n} \lambda_{d}
$$

The $\lambda_{n}$ are weights that are related to Selberg's sieve. Notice that we have

$$
a(1)=1
$$

and $a(n)=0$ for $1<n \leq z_{1}$. Moreover, for primes $p$, we have

$$
a(p)= \begin{cases}\frac{\log p / z_{1}}{\log z_{2} / z_{1}} & \text { if } z_{1}<p<z_{2} \\ 0 & \text { otherwise. }\end{cases}
$$

It was shown by Barban and Vehov [BaVe68] and Motohashi [Mo74] that

$$
\sum_{n \leq N}|a(n)|^{2} \ll \frac{N}{\log z_{2} / z_{1}} .
$$

Soon afterwards, S. Graham [Gr78] was able to prove the following asymptotic formulae: if $N \geq z_{2}$, then

$$
\sum_{n \leq N}|a(n)|^{2}=\frac{N}{\log z_{2} / z_{1}}+\mathbf{O}\left(\frac{N}{\left(\log z_{2} / z_{1}\right)^{2}}\right)
$$

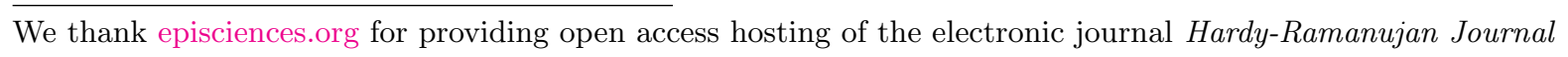


and if $z_{1} \leq N \leq z_{2}$, we have

$$
\sum_{n \leq N}|a(n)|^{2}=\frac{N \log N / z_{1}}{\left(\log z_{2} / z_{1}\right)^{2}}+\mathbf{O}\left(\frac{N}{\left(\log z_{2} / z_{1}\right)^{2}}\right) .
$$

This result has found significant applications to zero density theorems and to the estimation of Linnik's constant (see [Ju77] and [Gr81] for example).

The purpose of this note is to study the size of the sum when $n$ is constrained to range through a fixed arithmetic progression. In my joint work with R. Balasubramanian [BaMu92], we observed (Proposition 1.2) that for $N \geq r$ and $(b, r)=1$, we have

$$
\sum_{\substack{n \leq N \\ n \equiv b(\bmod r)}}|a(n)| \ll \frac{N}{\phi(r)^{\frac{1}{2}}\left(\log z_{2} / z_{1}\right)^{\frac{1}{2}}} .
$$

This follows immediately from Graham's result by the Cauchy-Schwarz inequality.

Soon after [BaMu92] was written, I worked out an asymptotic formula for the sum on the left by adapting Graham's methods. The result is that for $(b, r)=1$ and $N \geq r z_{2}^{2}$, we have

$$
\sum_{\substack{n \leq N \\ n \equiv b \bmod r}}|a(n)|^{2}=\frac{N}{\phi(r) \log z_{2} / z_{1}}+\mathbf{O}\left(\frac{N \sigma(r)}{\phi(r)^{2}\left(\log z_{2} / z_{1}\right)^{2}}\right) .
$$

If $r z_{1} z_{2} \leq N \leq r z_{2}^{2}$, then we show that

$$
\sum_{\substack{n \leq N \\ n \equiv b \bmod r}}|a(n)|^{2}=\frac{N}{\phi(r) \log z_{2} / z_{1}}+\mathbf{O}\left(\frac{N\left(\log r z_{2}^{2} / N\right)^{5}}{r\left(\log z_{2} / z_{1}\right)^{2}}\right)+\mathbf{O}\left(\frac{N(\log r)^{2}}{r\left(\log z_{2} / z_{1}\right)^{2}}\right)+\mathbf{O}\left(\frac{r z_{2}}{\left(\log z_{2} / z_{1}\right)^{2}}\right) .
$$

The last two terms on the right are not present if we have the additional condition $z_{1}>r$. Also, It will be clear from the arguments that the same methods will actually allow us to get estimates for $N<r z_{1} z_{2}$ as well but we do not pursue that here.

An immediate consequence of the first formula is a version of the Brun-Titchmarsh theorem in the following form. Denote by $\pi(N, r, b)$ the number of primes $\leq N$ which are $\equiv b \bmod r$. Then we have

We could try to use the second formula in a similar manner.

$$
\pi(N, r, b) \leq \frac{2 N}{\phi(r) \log N / r}+\mathbf{O}\left(\frac{N \sigma(r)}{\phi(r)^{2}(\log N / r)^{2}}\right) .
$$

Our method of proof for both formulae is elementary and uses only the usual prime number theorem. The proof of the first formula is a direct generalization of the work of Graham ([Gr78], §3) and represents the easy case.

Though these calculations were completed some years ago, I had not published them. However, the occasion of a volume honouring the memory of my friend S. Srinivasan caused me to look at them again. In particular, the consequence for the Brun-Titchmarsh theorem may still be of interest. When I was a Visiting Fellow at the Tata Institute for Fundamental Research in 1983-1984, Srinivasan was my office-mate and we shared many hours of mathematical conversation. We also enjoyed many social occasions together when we had a chance to discuss philosophical and even spiritual questions. Srinivasan was always a thorough and thoughtful individual and I look back on those occasions with many pleasant memories. Given that his main interest was in analytic number theory, I thought the topic of this article might have been of interest to him.

The note is organized as follows. In $\S 2$, we prove the first formula. In $\S 3$, we begin the proof of the second formula. In $\S 4$ and $\S 5$, we estimate certain error terms and in $\S 6$, we study the main term. Finally in $\S 7$, we complete the proof of the second formula.

I would like to thank the referees for helpful comments that helped to streamline the presentation. 


\section{The First Formula}

The purpose of this section is to prove the following.

Theorem 2.1. Suppose that $r z_{2}^{2} \leq N$ and $(b, r)=1$. Then,

$$
\sum_{\substack{n \leq N \\ n \equiv b}}|a(n)|^{2}=\frac{N}{\phi(r) \log z_{2} / z_{1}}+\mathbf{O}\left(\frac{N \sigma(r)}{\phi(r)^{2}\left(\log z_{2} / z_{1}\right)^{2}}\right) .
$$

The proof follows closely the method of [Gr78], $§ 3$ but we give the details in order to orient the reader. First, we note an immediate consequence. Denote by $\psi\left(N, z_{2}, r, b\right)$ the number of integers $n \leq N$ with $n \equiv b(\bmod r)$ all of whose prime factors are $\geq z_{2}$. Denote also by $\pi(N, r, b)$ the number of primes $p \leq N$ with $p \equiv b(\bmod r)$.

Corollary 2.2. If $r z_{2}^{2} \leq N$, then

$$
\psi\left(N, z_{2}, r, b\right) \leq \frac{N}{\phi(r) \log z_{2}}+\mathbf{O}\left(\frac{N \sigma(r)}{\phi(r)^{2}\left(\log z_{2}\right)^{2}}\right) .
$$

Proof. If $n \leq N$, and $n \equiv b(\bmod r)$ and it has all its prime divisors $\geq z_{2}$, then $a(n)=1$.

Corollary 2.3. We have

$$
\pi(N, r, b) \leq \frac{2 N}{\phi(r) \log N / r}+\mathbf{O}\left(\frac{N \sigma(r)}{\phi(r)^{2}(\log N / r)^{2}}\right)
$$

Proof. This follows on noting that

$$
\pi(N, r, b) \leq \pi\left(z_{2}, r, b\right)+\psi\left(N, z_{2}, r, b\right),
$$

and the trivial bound $\pi\left(z_{2}, r, b\right) \leq z_{2} / r+1$ and choosing $z_{2}=(N / r)^{\frac{1}{2}}$.

To prove the theorem, we notice that

$$
\left(\log z_{2} / z_{1}\right)^{2} \sum_{\substack{n \leq N \\ n \equiv b}}|a(n)|^{2}=\sum_{\substack{n \leq N \\ n \equiv b}}\left(\sum_{d \mid n} \Lambda_{1}(d)-\sum_{e \mid n} \Lambda_{2}(e)\right)^{2} .
$$

The right hand side is a sum of terms of the form

$$
S_{i, j}=\sum_{\substack{n \leq N \\ n \equiv b}} \sum_{\substack{(\bmod r) \\ d, e \mid n}} \Lambda_{i}(d) \Lambda_{j}(e) .
$$

where $i, j \in\{1,2\}$. The theorem will follow from the following.

Proposition 2.4. We have

$$
S_{i, j}=\frac{N}{\phi(r)} \log \min \left(z_{i}, z_{j}\right)+\mathbf{O}\left(z_{i} z_{j}\right)+\mathbf{O}\left(N \sigma(r) / \phi(r)^{2}\right) .
$$

In particular, if $r z_{i} z_{j} \leq N$, the first error term is $\mathbf{O}(N / r)$. 
Proof. By definition

$$
S_{i, j}=\sum \Lambda_{i}(d) \Lambda_{j}(e)\left\{\frac{N}{r[d, e]}+\mathbf{O}(1)\right\}
$$

The error term is

$$
\ll\left(\sum_{d \leq z_{i}}\left|\Lambda_{i}(d)\right|\right)\left(\sum_{e \leq z_{j}}\left|\Lambda_{j}(e)\right|\right) \ll z_{i} z_{j} .
$$

The main term is

$$
\frac{N}{r} \sum_{\substack{d \leq z_{i} \\ e \leq z_{j} \\(d, r)=(e, r)=1}} \frac{\Lambda_{i}(d) \Lambda_{j}(e)}{[d, e]} .
$$

Without loss of generality, we may suppose that $z_{i} \leq z_{j}$. We have

$$
\sum_{\substack{d \leq z_{i} \\ e \leq z_{j} \\(d, r)=(e, r)=1}} \frac{\Lambda_{i}(d) \Lambda_{j}(e)}{[d, e]}=\sum \frac{\Lambda_{i}(d) \Lambda_{j}(e)}{d e} \sum_{m \mid(d, e)} \phi(m)
$$

and inserting the definition of $\Lambda_{i}$ and $\Lambda_{j}$, the right hand side is seen to be

$$
\sum_{\substack{m \leq z_{i} \\(m, r)=1}} \frac{\mu(m)^{2} \phi(m)}{m^{2}}\left(\sum_{\substack{d_{0} \leq z_{i} / m \\\left(d_{0}, m r\right)=1}} \frac{\mu\left(d_{0}\right)}{d_{0}} \log \frac{z_{i}}{m d_{0}}\right)\left(\sum_{\substack{e_{0} \leq z_{j} / m \\\left(e_{0}, m r\right)=1}} \frac{\mu\left(e_{0}\right)}{e_{0}} \log \frac{z_{j}}{m e_{0}}\right) .
$$

We quote the following from [Gr78], §2:

Lemma 2.5. For any integer $a$ and any $c>0$, we have

$$
\sum_{\substack{n \leq Q \\(n, a)=1}} \frac{\mu(n)}{n} \log \frac{Q}{n}=\frac{a}{\phi(a)}+\mathbf{O}_{c}\left(\sigma_{-\frac{1}{2}}(a)(\log 2 Q)^{-c}\right) .
$$

Using this on the terms in parentheses, we find that the above is

$$
\sum_{\substack{m \leq z_{i} \\(m, r)=1}} \frac{\mu(m)^{2} \phi(m)}{m^{2}}\left\{\frac{m r}{\phi(m r)}+\mathbf{O}\left(\sigma_{-\frac{1}{2}}(m r)\left(\log 2 z_{i} / m\right)^{-2}\right\}^{2}\right.
$$

The error terms are $\mathbf{O}(1)$ just as in [Gr78], pp. 89-90. The main term is

$$
\frac{r^{2}}{\phi(r)^{2}} \sum_{\substack{m \leq z_{i} \\(m, r)=1}} \frac{\mu(m)^{2}}{\phi(m)} \text {. }
$$

This is seen to be

$$
\frac{r}{\phi(r)} \log z_{i}+\mathbf{O}\left(\frac{r \sigma(r)}{\phi(r)^{2}}\right)
$$




\section{The second formula}

The aim of the remaining sections is to prove the following asymptotic formula which will suffice to deduce the second formula.

Theorem 3.1. Suppose that $r z_{1} z_{2} \leq N \leq r z_{2}^{2}$. Then for $(b, r)=1$, we have

$$
\sum_{\substack{n \leq N \\ n \equiv b \bmod r}}\left(\sum_{d \mid n} \Lambda_{2}(d)\right)^{2}=\frac{N}{\phi(r)} \log z_{2}+\mathbf{O}\left(\frac{N}{r}\left(\log r z_{2}^{2} / N\right)^{5}\right)+\mathbf{O}\left(\frac{N}{r}(\log r)^{2}\right)+\mathbf{O}\left(r z_{2}\right) .
$$

Expanding the sum on the left, we have

$$
\sum_{\substack{n \leq N \\ n \equiv b \bmod r}}\left(\sum_{d \mid n} \Lambda_{2}(d)\right)\left(\sum_{e \mid n} \Lambda_{2}(e)\right)
$$

and we begin the proof by splitting this sum into three components

$$
S_{A}+S_{B}+\mathbf{O}\left(S_{C}\right)
$$

where in $S_{A}$ we restrict the sum to $d, e$ satisfying $[d, e]>N / r$. The remaining terms may be rearranged to give

$$
\sum_{[d, e] \leq N / r} \Lambda_{2}(d) \Lambda_{2}(e) \sum_{\substack{n \leq N \\ n=b \bmod r \\ n=0 \bmod [d, e]}} 1
$$

which is

$$
=\frac{N}{r} \sum_{\substack{[d, e] \leq N / r \\(d, r)=(e, r)=1}} \frac{\Lambda_{2}(d) \Lambda_{2}(e)}{[d, e]}+\mathbf{O}\left(\sum_{[d, e] \leq N / r}\left|\Lambda_{2}(d) \Lambda_{2}(e)\right|\right)
$$

which we write as

$$
S_{B}+\mathbf{O}\left(S_{C}\right)
$$

In $\S 4$., we shall show that

$$
S_{A} \ll \frac{N}{r}\left(\log r z_{2}^{2} / N\right)^{5}+\frac{N}{r}(\log r)^{2}+r z_{2} .
$$

In $\S 5$., we shall show that

$$
S_{C} \ll \frac{N}{r}\left(\log r z_{2}^{2} / N\right)^{4} .
$$

Finally, in $\S 6$., we shall deal with the main term $S_{B}$, and in $\S 7$. we collect together the various pieces to complete the proof of Theorem 3.1.

We state explicitly the consequence of Theorem 3.1 for the $a(n)$.

Theorem 3.2. Suppose that $r z_{2}^{2} \geq N \geq r z_{1} z_{2}$. Then

$$
\sum_{\substack{n \leq N \\ n \equiv b(\bmod r)}}|a(n)|^{2}=\frac{N}{\phi(r) \log z_{2} / z_{1}}+\mathbf{O}\left(\frac{N\left(\log r z_{2}^{2} / N\right)^{5}}{r\left(\log z_{2} / z_{1}\right)^{2}}\right)+\mathbf{O}\left(\frac{N(\log r)^{2}}{r\left(\log z_{2} / z_{1}\right)^{2}}\right)+\mathbf{O}\left(\frac{r z_{2}}{\left(\log z_{2} / z_{1}\right)^{2}}\right) .
$$




\section{Estimation of $S_{A}$}

Proposition 4.1. Suppose that $r z_{2}^{2} \geq N>r$. Then

$$
\sum_{\substack{n \leq N \\ n \equiv b}} \sum_{\substack{[d, e] \mid n \\[\bmod r, e]>N / r}}\left|\Lambda_{2}(d) \Lambda_{2}(e)\right| \ll \frac{N}{r}\left(\log r z_{2}^{2} / N\right)^{5}+\frac{N}{r}(\log r)^{2}+r z_{2} .
$$

Remark 4.2. Our argument will show that in certain ranges of $r, z_{i}, z_{j}$ and $N$, this estimate can be refined.

Proof. Write $\rho=(d, e)$. Then, each $n$ can be written as $n=\rho e_{0} d_{0} n_{0}$ with $d=\rho d_{0}, e=\rho e_{0}$ and $[d, e]=\rho d_{0} e_{0}>N / r$. This last condition implies that $n_{0}<r$. The sum over $e_{0}$ then ranges over the interval

In order for this to be nonempty, we need

$$
\frac{N}{r \rho d_{0}}<e_{0} \leq \frac{z_{2}}{\rho}
$$

$$
d_{0}>N / r z_{2} .
$$

But $d_{0} \leq z_{2} / \rho$ and so $\rho<r z_{2}^{2} / N$. Thus, our sum is

$$
\sum_{\substack{\rho<r z_{2}^{2} / N \\(\rho, r)=1}} \sum_{\substack{n_{0}<r \\\left(n_{0}, r\right)=1}} \sum_{\substack{\left.\frac{N}{r \rho}<d_{0} e_{0} \leq \frac{N}{\rho n_{0}} \\ d_{0} e_{0} \equiv \bar{p} 0_{0} b \\ d_{0}<z_{2} / \rho, e_{0}<z_{2} / \rho\right)}}\left|\Lambda_{2}\left(d_{0} \rho\right) \Lambda_{2}\left(e_{0} \rho\right)\right| .
$$

Here, for any residue class $\beta(\bmod r)($ with $(\beta, r)=1)$, we are writing $\bar{\beta}(\bmod r)$ for the inverse class. The inner sum is

$$
\sum_{\substack{\frac{N}{r \rho}<d_{0} e_{0} \leq \frac{N}{\rho n_{0}} \\ d_{0} e_{0} \equiv \overline{\rho n_{0} b}(\bmod r)}}\left|\mu\left(d_{0} \rho\right)\left(\log z_{2} / d_{0} \rho\right) \mu\left(e_{0} \rho\right)\left(\log z_{2} / e_{0} \rho\right)\right| .
$$

Separating the $d_{0}$ and the $e_{0}$ sums, we find that this is

$$
\sum_{d_{0}}\left|\mu\left(d_{0} \rho\right) \log \frac{z_{2}}{d_{0} \rho}\right| \sum_{e_{0}}\left|\mu\left(e_{0} \rho\right) \log \frac{z_{2}}{e_{0} \rho}\right|
$$

where the sum over $d_{0}$ is in the range

$$
\frac{N}{r z_{2}}<d_{0}<\min \left(\frac{z_{2}}{\rho}, \frac{N}{n_{0} \rho}\right)
$$

and the sum over $e_{0}$ is in the range

$$
\frac{N}{r d_{0} \rho}<e_{0}<\min \left(\frac{z_{2}}{\rho}, \frac{N}{n_{0} d_{0} \rho}\right)
$$

with the additional condition

$$
e_{0} \equiv b \cdot \overline{d_{0} \rho n_{0}} \quad(\bmod r)
$$

First, consider the contribution of the $e_{0}$ which satisfy $e_{0}>r$. Writing $e_{0}=*+e_{1} r$, we see that the inner sum is

$$
\leq \sum_{1 \leq e_{1} \leq \min \left(\frac{N}{n_{0} \rho d_{0} r}, \frac{z_{2}}{r \rho}\right)} \log \frac{z_{2}}{e_{1} r \rho} .
$$




\section{A. Case 1}

If $N / n_{0} d_{0} \leq z_{2}$, then this is

$$
\ll \frac{N}{n_{0} \rho d_{0} r}\left(\log \frac{z_{2} n_{0} d_{0}}{N}+1\right) .
$$

Inserting this into the sum over $d_{0}$, we find that it is

$$
\ll \frac{N}{n_{0} \rho r} \sum_{\frac{N}{z_{2} n_{0}}<d_{0} \leq \frac{z_{2}}{\rho}} \frac{1}{d_{0}}\left(\log \frac{z_{2}}{d_{0} \rho}\right)\left(\log \frac{z_{2} n_{0} d_{0}}{N}+1\right) .
$$

For this sum to be nonempty, we require

$$
\rho<z_{2}^{2} n_{0} / N
$$

Using the following consequence of the arithmetic mean - geometric mean inequality,

$$
(\log A)(\log B) \leq(\log A B)^{2}
$$

for $A, B \geq 1$, the above sum is

$$
\ll \frac{N}{n_{0} \rho r} \sum \frac{1}{d_{0}}\left(\log \frac{z_{2}^{2} n_{0}}{N \rho}\right)^{2} .
$$

The sum over $d_{0}$ is

$$
\ll \log \frac{z_{2}^{2} n_{0}}{N \rho}
$$

and so, we have to estimate

$$
\sum_{n_{0}<r} \sum_{\rho<n_{0} z_{2}^{2} / N} \frac{N}{r n_{0} \rho}\left(\log \frac{z_{2}^{2} n_{0}}{N \rho}\right)^{3}
$$

and this is

$$
\ll \frac{N}{r} \sum_{N / z_{2}^{2} \leq n_{0}<r} \frac{1}{n_{0}}\left(\log \frac{z_{2}^{2} n_{0}}{N}\right)^{4}
$$

which in turn is

$$
\ll \frac{N}{r}\left(\log \frac{r z_{2}^{2}}{N}\right)^{5} \text {. }
$$

\section{B. Case 2}

If $N / n_{0} d_{0} \geq z_{2}$, the sum in (4.1) is

$$
\ll \sum_{e_{1} \leq z_{2} / r \rho} \log \frac{z_{2}}{e_{1} r \rho} \ll \frac{z_{2}}{r \rho} .
$$

Notice that in order for such terms to exist, we need $\rho \leq z_{2} / r$ and in particular, $r \leq z_{2}$. Inserting this estimate into the sum over $d_{0}$, we get

$$
\frac{z_{2}}{r \rho} \sum_{d_{0}<\min \left(N / n_{0} z_{2}, z_{2} / \rho\right)} \log \frac{z_{2}}{d_{0} \rho} .
$$

We distinguish two sub cases. 


\section{C. Case 2(a)}

Suppose that

$$
z_{2} / \rho<N / n_{0} z_{2} .
$$

Then, the sum over $d_{0}$ is $\mathbf{O}\left(z_{2} / \rho\right)$ and so the overall contribution is

$$
\ll \sum_{\rho<r z_{2}^{2} / N} \sum_{\substack{n_{0}<N \rho / z_{2}^{2} \\ n_{0}<r}} \frac{z_{2}^{2}}{r \rho^{2}} .
$$

This simplifies to

$$
\frac{z_{2}^{2}}{r} \sum_{\rho<r z_{2}^{2} / N} \frac{1}{\rho^{2}} \frac{N \rho}{z_{2}^{2}}
$$

and this is

$$
\ll \frac{N}{r} \log \frac{r z_{2}^{2}}{N}
$$

\section{D. Case 2(b)}

Consider the remaining case

$$
z_{2} / \rho \geq N / n_{0} z_{2}
$$

Then, the sum over $d_{0}$ is

$$
\ll \frac{N}{n_{0} z_{2}} \log \left(\frac{n_{0} z_{2}^{2}}{\rho N}\right)
$$

and so the overall contribution is

$$
\ll \sum_{n_{0}<r} \sum_{\rho<\min \left(n_{0} z_{2}^{2} / N, z_{2} / r\right)} \frac{z_{2}}{\rho r} \frac{N}{n_{0} z_{2}}\left(\log \frac{n_{0} z_{2}^{2}}{\rho N}\right)
$$

which is

$$
=\frac{N}{r} \sum_{n_{0}<r} \frac{1}{n_{0}} \sum_{\rho} \frac{1}{\rho}\left(\log \frac{n_{0} z_{2}^{2}}{\rho N}\right) .
$$

This sum can be split into two subsums, the first of which is

$$
\frac{N}{r} \sum_{n_{0}<\min \left(r, N / r z_{2}\right)} \frac{1}{n_{0}} \sum_{\rho<n_{0} z_{2}^{2} / N} \frac{1}{\rho}\left(\log \frac{n_{0} z_{2}^{2}}{\rho N}\right)
$$

and this is

$$
\ll \frac{N}{r} \sum_{N / z_{2}^{2}<n_{0}<r} \frac{1}{n_{0}}\left(\log \frac{n_{0} z_{2}^{2}}{N}\right)^{2}
$$

which is

$$
\ll \frac{N}{r}\left(\log \frac{r z_{2}^{2}}{N}\right)^{3} .
$$

The second is

$$
\frac{N}{r} \sum_{\rho<z_{2} / r} \frac{1}{\rho} \sum_{\substack{N / r z_{2}<n_{0}<r \\ \rho N / z_{2}^{2}<n_{0}}} \frac{1}{n_{0}}\left(\log \frac{n_{0} z_{2}^{2}}{\rho N}\right)
$$


which is seen to be

$$
\ll \frac{N}{r} \sum_{\rho<r z_{2}^{2} / N} \frac{1}{\rho}\left(\log \frac{r z_{2}^{2}}{N}\right)^{2}
$$

and this is

$$
\ll \frac{N}{r}\left(\log \frac{r z_{2}^{2}}{N}\right)^{3} .
$$

Note that this term is present only if $r^{2} z_{2}>N$.

To summarize, Case (2) occurs only if $r<z_{2}$ and in this case, it contributes

$$
\frac{N}{r}\left(\log \frac{r z_{2}^{2}}{N}\right)^{3} \text {. }
$$

\section{E. The contribution of terms with $e_{0}<r$}

By interchanging the roles of $e_{0}$ and $d_{0}$, we may also suppose that $d_{0}<r$. We see that as $n_{0}<r$, the congruence condition

$$
e_{0} d_{0} \rho n_{0} \equiv b \quad(\bmod r)
$$

implies that $e_{0}, d_{0}, \rho$ uniquely determine $n_{0}$. Thus, our sum is

$$
\leq \sum_{d_{0}, e_{0}} \sum_{\rho}\left(\log \frac{z_{2}}{d_{0} \rho}\right)\left(\log \frac{z_{2}}{e_{0} \rho}\right) .
$$

Here, the outer sum ranges over $d_{0}, e_{0}<r$ satisfying $N / r z_{2}<d_{0}<z_{2}$ and $e_{0}<z_{2}$ and the inner sum ranges over $\rho$ satisfying

$$
\frac{N}{r d_{0} e_{0}} \leq \rho \leq \min \left(\frac{z_{2}}{d_{0}}, \frac{z_{2}}{e_{0}}, \frac{r z_{2}^{2}}{N}\right)
$$

Since

$$
\frac{N}{r z_{2}}<d_{0}
$$

we see that

$$
\frac{z_{2}}{d_{0}} \leq \frac{r z_{2}^{2}}{N}
$$

Also,

$$
e_{0} \geq \frac{N}{r \rho d_{0}}>\frac{N}{r z_{2}}
$$

and so

$$
\frac{z_{2}}{e_{0}} \leq \frac{r z_{2}^{2}}{N}
$$

Let us set

$$
w=\min \left(\frac{z_{2}}{d_{0}}, \frac{z_{2}}{e_{0}}\right) .
$$

We will consider the case $w=z_{2} / d_{0}$, the other case being similar. In this case we must have

$$
r z_{2} \leq N
$$

It forces the condition

$$
\frac{N}{r z_{2}} \leq e_{0} \leq d_{0}
$$


Using the identity (4.2), the sum over $\rho$ is

$$
\ll \frac{N}{r d_{0} e_{0}} \log \left(\frac{N^{2}}{r^{2} z_{2}^{2} d_{0} e_{0}}\right)+\frac{z_{2}}{d_{0}} \log \frac{d_{0}}{e_{0}} .
$$

Now we insert this into the sum over $d_{0}$ and $e_{0}$. For the $e_{0}$ sum to be nonempty, we must also have $r^{2} z_{2} \geq N$ (since $r^{2} z_{2} \geq r z_{2} d_{0} \geq N$ ). In this case, the $e_{0}$ sum is

$$
\ll \frac{N}{r d_{0}} \log \frac{N}{r z_{2}}+z_{2} .
$$

Summing this over $d_{0}$, we get an estimate of

$$
\ll \frac{N}{r}\left(\log \frac{N}{r z_{2}}\right)(\log r)+r z_{2}
$$

and this is

$$
\leq \frac{N}{r}(\log r)^{2}+r z_{2}
$$

\section{Estimation of $S_{C}$}

Proposition 5.1. Suppose that $r z_{i} z_{j} \geq N>r$. Then, we have

$$
\sum_{[d, e] \leq N / r}\left|\Lambda_{i}(d) \Lambda_{j}(e)\right| \ll \frac{N}{r}\left(\log \frac{r z_{i} z_{j}}{N}\right)^{4} .
$$

Proof. Set $\rho=(d, e)$. Thus, the sum is

$$
\sum_{d e \leq N \rho / r}\left|\Lambda_{i}(d) \Lambda_{j}(e)\right|=\sum_{d}\left|\Lambda_{i}(d)\right| \sum_{\substack { \rho \mid d \\
\begin{subarray}{c}{e \leq N \rho / r d \\
\rho / \rho \\
(e / \rho, d / \rho)=1{ \rho | d \\
\begin{subarray} { c } { e \leq N \rho / r d \\
\rho / \rho \\
( e / \rho , d / \rho ) = 1 } }\end{subarray}}\left|\Lambda_{j}(e)\right| .
$$

Write $d=d_{0} \rho$ and $e=e_{0} \rho$. Then the above is

$$
\sum_{\rho \leq z_{i}} \sum_{d_{0} \leq z_{i} / \rho}\left|\Lambda_{i}\left(d_{0} \rho\right)\right| \sum_{\substack{e_{0} \leq N / r d \\\left(e_{0}, d_{0}\right)=1}}\left|\Lambda_{j}\left(e_{0} \rho\right)\right| .
$$

In the inner sum, we need in fact that

$$
e_{0} \leq \min \left(\frac{N}{r d}, \frac{z_{j}}{\rho}\right)
$$

Consider the contribution of terms with

$$
z_{j} \leq N / r d_{0}
$$

We have to estimate

$$
\sum_{\rho \leq z_{i}} \sum_{d_{0} \leq \min \left(z_{i} / \rho, N / r z_{j}\right)}\left(\log \frac{z_{i}}{d_{0} \rho}\right) \sum_{\substack{e_{0} \leq z_{j} / \rho \\\left(e_{0}, d_{0}\right)=1}}\left(\log \frac{z_{j}}{e_{0} \rho}\right)
$$


We see that

$$
\frac{z_{i}}{\rho}<\frac{N}{r z_{j}}
$$

holds if and only if

$$
\rho>\frac{r z_{i} z_{j}}{N}
$$

In this case, the sum is

$$
\sum_{\rho>r z_{i} z_{j} / N} \sum_{d_{0} \leq z_{i} / \rho}\left(\log \frac{z_{i}}{d_{0} \rho}\right) \sum_{e_{0} \leq z_{j} / \rho}\left(\log \frac{z_{j}}{e_{0} \rho}\right)
$$

and this is

$$
\ll \sum_{\rho>r z_{i} z_{j} / N} \frac{z_{i} z_{j}}{\rho^{2}} \ll z_{i} z_{j} \frac{N}{r z_{i} z_{j}}=\frac{N}{r} .
$$

The contribution of the remaining terms (the one that do not satisfy (5.4)) is

$$
\sum_{\rho \leq r z_{i} z_{j} / N} \sum_{d_{0} \leq N / r z_{j}}\left(\log \frac{z_{i}}{d_{0} \rho}\right) \sum_{e_{0} \leq z_{j} / \rho}\left(\log \frac{z_{j}}{e_{0} \rho}\right) .
$$

This is seen to be

$$
\ll \sum_{\rho \leq r z_{i} z_{j} / N} \frac{z_{j}}{\rho}\left(\frac{N}{r z_{j}}\left(\log \frac{z_{i}}{\rho} \frac{r z_{j}}{N}\right)+\mathbf{O}\left(\frac{N}{r z_{j}}\right)\right)
$$

Simplifying, this is

$$
\ll \frac{N}{r} \sum_{\rho<r z_{i} z_{j} / N} \frac{1}{\rho}\left(\log \frac{r z_{i} z_{j}}{N \rho}\right)+\mathbf{O}\left(\frac{N}{r} \sum_{\rho<r z_{i} z_{j} / N} \frac{1}{\rho}\right)
$$

which is

$$
\ll \frac{N}{r}\left(\log \frac{r z_{i} z_{j}}{N}\right)^{2}
$$

Consider now the case that

$$
z_{j}>N / r d_{0}
$$

Note that

$$
\frac{z_{i}}{\rho} \geq d_{0}>\frac{N}{r z_{j}} .
$$

Thus, $\rho<r z_{i} z_{j} / N$. We have

$$
\sum_{\rho \leq \min \left(z_{i}, r z_{i} z_{j} / N\right)} \sum_{\frac{N}{r z_{j}}<d_{0} \leq z_{i} / \rho}\left(\log \frac{z_{i}}{d_{0} \rho}\right) \sum_{e_{0} \leq N / r d_{0} \rho}\left(\log \frac{z_{j}}{e_{0} \rho}\right) .
$$

The sum over $e_{0}$ is

$$
\frac{N}{r d_{0} \rho}\left(\log \left(\frac{z_{j}}{\rho} \frac{r d_{0} \rho}{N}\right)+\mathbf{O}(1)\right) .
$$

Inserting this, we get that our sum is

$$
\ll \frac{N}{r} \sum_{\rho \leq \min \left(z_{i}, r z_{i} z_{j} / N\right)} \frac{1}{\rho} \sum_{\frac{N}{r z_{j}}<d_{0} \leq z_{i} / \rho} \frac{1}{d_{0}}\left(\log \frac{z_{i}}{d_{0} \rho}\right)\left(\log \frac{r d_{0} z_{j}}{N}\right) .
$$


The sum over $d_{0}$ is easily seen to be

$$
\sim \frac{1}{6}\left(\log \frac{r z_{i} z_{j}}{N \rho}\right)^{3}
$$

Inserting this, we find that our sum is

$$
\ll \frac{N}{r} \sum_{\rho \leq \min \left(z_{i}, r z_{i} z_{j} / N\right)} \frac{1}{\rho}\left(\log \frac{r z_{i} z_{j}}{N \rho}\right)^{3} \ll \frac{N}{r}\left(\log \frac{r z_{i} z_{j}}{N}\right)^{4} .
$$

\section{The main term}

Finally, we deal with the main term. We need the following technical result.

Proposition 6.1. Suppose that $r z_{i} z_{j} \geq N>r$. Then, we have

$$
\begin{gathered}
\sum_{\substack{\left[a_{1}, a_{2}\right] \leq M \\
\left(a_{1}, r_{1}\right)=\left(a_{2}, r_{2}\right)=1}} \frac{\Lambda_{i}\left(d_{1} a_{1}\right) \Lambda_{j}\left(d_{2} a_{2}\right)}{a_{1} a_{2}}=\mu\left(d_{1}\right) \mu\left(d_{2}\right) \frac{d_{1} r_{1}}{\phi\left(d_{1} r_{1}\right)} \frac{d_{2} r_{2}}{\phi\left(d_{2} r_{2}\right)} \sum_{\gamma} \frac{\mu(\gamma)^{2}}{\phi(\gamma)^{2}}+ \\
+\mathbf{O}\left(\left(\log \frac{z_{i} z_{j}}{d_{1} d_{2} M}\right)^{4}\right)+E
\end{gathered}
$$

where $\gamma$ ranges over

$$
\gamma \leq \min \left(\frac{z_{i}}{d_{1}}, \frac{z_{j}}{d_{2}}\right)
$$

and

$$
\left(\gamma, r_{1} r_{2} d_{1} d_{2}\right)=1
$$

Here,

$$
\begin{gathered}
E \ll \frac{d_{1} r_{1}}{\phi\left(d_{1} r_{1}\right)} \sigma_{-\frac{1}{2}}\left(d_{2} r_{2}\right)\left(\log 2 z_{j} / d_{2}\right)^{-4} \\
+\frac{d_{2} r_{2}}{\phi\left(d_{2} r_{2}\right)} \sigma_{-\frac{1}{2}}\left(d_{1} r_{1}\right)\left(\log 2 z_{i} / d_{1}\right)^{-4} \\
+\sigma_{-\frac{1}{2}}\left(d_{1} r_{1}\right) \sigma_{-\frac{1}{2}}\left(d_{2} r_{2}\right)\left(\log 2 z_{i} / d_{1}\right)^{-4}\left(\log 2 z_{j} / d_{2}\right)^{-4} .
\end{gathered}
$$

Proof. Write $\gamma=\left(a_{1}, a_{2}\right)$. The condition $\left[a_{1}, a_{2}\right] \leq M$ is then $a_{1} a_{2} \leq M \gamma$. Write

$$
a_{1}=\gamma a_{1}^{\prime}, \quad a_{2}=\gamma a_{2}^{\prime} .
$$

Then, our sum is

$$
\sum_{\left(\gamma, r_{1} r_{2}\right)=1} \sum_{\left(a_{1}^{\prime}, r_{1}\right)=1} \frac{\Lambda_{i}\left(d_{1} \gamma a_{1}^{\prime}\right)}{\gamma a_{1}^{\prime}} \sum_{\substack{\left(a_{2}^{\prime}, r_{2}\right)=1 \\ a_{2}^{\prime} \leq M / \gamma a_{1}^{\prime}}} \frac{\Lambda_{j}\left(d_{2} \gamma a_{2}^{\prime}\right)}{\gamma a_{2}^{\prime}}
$$

since $a_{1} a_{2} \leq M \gamma$ means that $\gamma a_{1}^{\prime} a_{2}^{\prime} \leq M$. Moreover, we may as well assume that

$$
\left(a_{1}, d_{1}\right)=\left(a_{2}, d_{2}\right)=1
$$

Thus, we can rewrite our sum as

$$
\sum_{\left(\gamma, r_{1} r_{2} d_{1} d_{2}\right)=1} \frac{\mu\left(d_{1} \gamma\right) \mu\left(d_{2} \gamma\right)}{\gamma^{2}} \sum_{\substack{\left(a_{1}^{\prime}, d_{1} \gamma r_{1}\right)=1 \\ a_{1}^{\prime} \leq \min \left(M / \gamma, z_{i} / d_{1} \gamma\right)}} \frac{\mu\left(a_{1}^{\prime}\right) \log z_{i} / d_{1} \gamma a_{1}^{\prime}}{a_{1}^{\prime}} \times \sum_{\substack{\left(a_{2}^{\prime}, d_{2} \gamma r_{2}\right)=1 \\ a_{2}^{\prime} \leq \min \left(M / \gamma a_{1}^{\prime}, z_{j} / d_{2} \gamma\right)}} \frac{\mu\left(a_{2}^{\prime}\right) \log z_{j} / d_{2} \gamma a_{2}^{\prime}}{a_{2}^{\prime}} .
$$


Suppose that $a_{1}^{\prime} \geq M d_{2} / z_{j}$. Then, the innermost sum is (using Lemma 2.5 quoted in $\S 2$ )

$$
\frac{d_{2} \gamma r_{2}}{\phi\left(d_{2} \gamma r_{2}\right)}+\mathbf{O}\left(\sigma_{-\frac{1}{2}}\left(d_{2} \gamma r_{2}\right)\left(\log 2 z_{j} / d_{2} \gamma\right)^{-4}\right)+\mathbf{O}\left(\left(\log \frac{z_{j} a_{1}^{\prime}}{d_{2} M}\right)^{2}\right)
$$

where the last term is present only if

$$
\frac{M}{a_{1}^{\prime}}<\frac{z_{j}}{d_{2}}
$$

The contribution of the last term above, when inserted into the $a_{1}^{\prime}$ sum is

$$
\ll \sum_{a_{1}^{\prime}} \frac{1}{a_{1}^{\prime}}\left(\log \frac{z_{i}}{d_{1} \gamma a_{1}^{\prime}}\right)\left(\log \frac{z_{j} a_{1}^{\prime}}{d_{2} M}\right)^{2}
$$

where the range of the sum is

$$
\frac{M d_{2}}{z_{j}} \leq a_{1}^{\prime} \leq \frac{z_{i}}{d_{1} \gamma}
$$

We see that it is

$$
\ll\left(\log \frac{z_{i} / d_{1} \gamma}{d_{2} M / z_{j}}\right)^{4}=\left(\log \frac{z_{i} z_{j}}{d_{1} d_{2} \gamma M}\right)^{4} .
$$

Inserting this into the $\gamma$ sum yields an error term of

$$
\ll \sum_{\gamma} \frac{1}{\gamma^{2}}\left(\log \frac{z_{i} z_{j}}{d_{1} d_{2} \gamma M}\right)^{4} \ll\left(\log \frac{z_{i} z_{j}}{d_{1} d_{2} M}\right)^{4} .
$$

We are left with the problem of estimating

$$
\sum_{\left(\gamma, r_{1} r_{2}\right)=1} \frac{\mu\left(d_{1} \gamma\right) \mu\left(d_{2} \gamma\right)}{\gamma^{2}}\left(\sum_{\left(a_{1}^{\prime}, d_{1} \gamma r_{1}\right)=1} \frac{\mu\left(a_{1}^{\prime}\right) \log z_{i} / d_{1} \gamma a_{1}^{\prime}}{a_{1}^{\prime}}\left(\frac{d_{2} \gamma r_{2}}{\phi\left(d_{2} \gamma r_{2}\right)}+\mathbf{O}\left(\sigma_{-\frac{1}{2}}\left(d_{2} \gamma r_{2}\right)\left(\log 2 z_{j} / d_{2} \gamma\right)^{-4}\right)\right)\right) .
$$

The sum over $a_{1}^{\prime}$ can also be estimated using the Lemma 2.5. It is equal to

$$
\frac{d_{1} \gamma r_{1}}{\phi\left(d_{1} \gamma r_{1}\right)}+\mathbf{O}\left(\sigma_{-\frac{1}{2}}\left(d_{1} \gamma r_{1}\right)\left(\log 2 z_{i} / d_{1} \gamma\right)^{-4}\right)
$$

Inserting this, we find that the main terms give

$$
\sum_{\left(\gamma, r_{1} r_{2} d_{1} d_{2}\right)=1} \frac{\mu\left(d_{1} \gamma\right) \mu\left(d_{2} \gamma\right)}{\gamma^{2}} \frac{d_{1} d_{2} r_{1} r_{2} \gamma^{2}}{\phi\left(d_{1} \gamma r_{1}\right) \phi\left(d_{2} \gamma r_{2}\right)}
$$

which is equal to

$$
\mu\left(d_{1}\right) \mu\left(d_{2}\right) \frac{d_{1} r_{1}}{\phi\left(d_{1} r_{1}\right)} \frac{d_{2} r_{2}}{\phi\left(d_{2} r_{2}\right)} \sum_{\left(\gamma, r_{1} r_{2} d_{1} d_{2}\right)=1} \frac{\mu(\gamma)^{2}}{\phi(\gamma)^{2}} .
$$

The sum over $\gamma$ extends to

$$
\gamma \leq \min \left(\frac{z_{i}}{d_{1}}, \frac{z_{j}}{d_{2}}\right)
$$

Now we consider the cross terms. There are three of them. The first is

$$
\ll \sum_{\left(\gamma, r_{1} r_{2} d_{1} d_{2}\right)=1} \frac{1}{\gamma^{2}} \frac{d_{1} \gamma r_{1}}{\phi\left(d_{1} \gamma r_{1}\right)} \sigma_{-\frac{1}{2}}\left(d_{2} \gamma r_{2}\right)\left(\log 2 z_{j} / d_{2} \gamma\right)^{-4}
$$


which is

$$
\ll \frac{d_{1} r_{1}}{\phi\left(d_{1} r_{1}\right)} \sigma_{-\frac{1}{2}}\left(d_{2} r_{2}\right)\left(\log 2 z_{j} / d_{2}\right)^{-4}
$$

Similarly, the second is

$$
\ll \frac{d_{2} r_{2}}{\phi\left(d_{2} r_{2}\right)} \sigma_{-\frac{1}{2}}\left(d_{1} r_{1}\right)\left(\log 2 z_{i} / d_{1}\right)^{-4}
$$

and the third is

$$
\ll \sigma_{-\frac{1}{2}}\left(d_{1} r_{1}\right) \sigma_{-\frac{1}{2}}\left(d_{2} r_{2}\right)\left(\log 2 z_{i} / d_{1}\right)^{-4}\left(\log 2 z_{j} / d_{2}\right)^{-4} .
$$

This proves the result.

We only need to apply this result in the following case.

Proposition 6.2. Suppose that $r z_{2}^{2} \geq N>r$. Then, we have

$$
\sum_{\substack{[d, e] \leq N / r \\(d, r)=(e, r)=1}} \frac{\Lambda_{2}(d) \Lambda_{2}(e)}{[d, e]}=\frac{r}{\phi(r)} \log z_{2}+\mathbf{O}\left(\left(\log \frac{r z_{2}^{2}}{N}\right)^{5}\right)+\mathbf{O}\left(\frac{r}{\phi(r)} \sigma_{-\frac{1}{2}}(r)\left(\log 2 z_{2}\right)^{-4}\right) .
$$

Proof. Let us set $\rho=(d, e)$. Then the sum in question may be written as

$$
\sum_{\substack{[d, e] \leq N / r \\(d, r)=(e, r)=1}} \frac{\Lambda_{2}(d) \Lambda_{2}(e)}{d e} \sum_{u \mid \rho} \phi(u)=\sum_{\substack{u \leq z_{2} \\(u, r)=1}} \frac{\phi(u)}{u^{2}} \sum_{\substack{\left[d_{1}, e_{1}\right] \leq N / r u \\\left(d_{1}, r\right)=\left(e_{1}, r\right)=1}} \frac{\Lambda_{2}\left(u d_{1}\right) \Lambda_{2}\left(u e_{1}\right)}{d_{1} e_{1}} .
$$

Applying Proposition 5.1 to the inner sum, we find that the above is

$$
\sum_{\substack{u \leq z_{2} \\(u, r)=1}} \frac{\phi(u)}{u^{2}}\left(\mu(u)^{2}\left(\frac{u r}{\phi(u r)}\right)^{2} \sum_{\substack{\left(\gamma_{1}, u r\right)=1 \\ \gamma_{1} \leq z_{2} / u}} \frac{\mu\left(\gamma_{1}\right)^{2}}{\phi\left(\gamma_{1}\right)^{2}}+E\right)
$$

where

$$
\begin{gathered}
E=\mathbf{O}\left(\left(\frac{u r}{\phi(u r)}\right)^{2} \frac{\left(\log \log z_{2}\right)^{2}}{z_{2}}\right)+\mathbf{O}\left(\frac{u r}{\phi(u r)} \sigma_{-\frac{1}{2}}(u r)\left(\log 2 z_{2} / u\right)^{-4}\right) \\
+\mathbf{O}\left(\sigma_{-\frac{1}{2}}(u r)^{2}\left(\log 2 z_{2} / u\right)^{-8}\right)+\mathbf{O}\left(\left(\log \frac{r z_{2}^{2}}{u N}\right)^{4}\right) .
\end{gathered}
$$

This is

$$
\sum_{\substack{u \leq z_{2} \\(u, r)=1}} \frac{\mu(u)^{2}}{\phi(u)} \frac{r^{2}}{\phi(r)^{2}} \sum_{\substack{\left(\gamma_{1}, u r\right)=1 \\ \gamma_{1} \leq z_{2} / u}} \frac{\mu\left(\gamma_{1}\right)^{2}}{\phi\left(\gamma_{1}\right)^{2}}+\mathbf{O}\left(\frac{r}{\phi(r)} \sigma_{-\frac{1}{2}}(r)\left(\log 2 z_{2}\right)^{-4}\right)+\mathbf{O}\left(\left(\log \frac{r z_{2}^{2}}{N}\right)^{5}\right) .
$$

The main term is

$$
\frac{r^{2}}{\phi(r)^{2}}\left(\sum_{\gamma=1}^{\infty} \frac{\mu(\gamma)^{2}}{\phi(\gamma)^{2}}\right) \sum_{\substack{u \leq z_{2} \\(u, r)=1}} \frac{\mu(u)^{2}}{\phi(u)} \prod_{p \mid u r}\left(1+\frac{1}{(p-1)^{2}}\right)^{-1}+\mathbf{O}\left(\frac{r^{2}}{\phi(r)^{2}} \log \log z_{2}\right) .
$$




\section{7. $\quad$ Proof of Theorems 3.1 and 3.2}

As described in $\S 3$, we have written

$$
\sum_{\substack{n \leq N \\ n \equiv b}}\left(\sum_{d \mid n} \Lambda_{2}(d)\right)\left(\sum_{e \mid n} \Lambda_{2}(e)\right)=S_{A}+S_{B}+\mathbf{O}\left(S_{C}\right),
$$

where in $S_{A}$, we require that $[d, e]>N / r$.

By Proposition 4.1, we deduce that

$$
S_{A} \ll \frac{N}{r}\left(\log \frac{r z_{2}^{2}}{N}\right)^{5}+\frac{N}{r}(\log r)^{2}+r z_{2} .
$$

As for $S_{C}$, we have by Proposition 5.1 that

$$
S_{C} \ll \frac{N}{r}\left(\log \frac{r z_{2}^{2}}{N}\right)^{4} .
$$

By Proposition 6.2, we have

$$
S_{B}=\frac{N}{r}\left(\frac{r}{\phi(r)} \log z_{2}+\mathbf{O}\left(\frac{r}{\phi(r)} \sigma_{-\frac{1}{2}}(r)\left(\log 2 z_{2}\right)^{-4}\right)+\mathbf{O}\left(\left(\log r z_{2}^{2} / N\right)^{5}\right)\right) .
$$

This proves Theorem 3.1.

For Theorem 3.2, as in $\S 2$, we have to estimate three sums of the form

$$
S_{i, j}=\sum_{\substack{n \leq N \\ n \equiv b}}\left(\sum_{d \mid n} \Lambda_{i}(d)\right)\left(\sum_{e \mid n} \Lambda_{j}(e)\right) .
$$

For the cases $i=j=1$ and $i=1, j=2$, the condition $N \geq r z_{i} z_{j}$ is satisfied and so we get the desired estimate from Proposition 1. The only remaining case is $i=j=2$ where this condition is not satisfied. This case follows from Theorem 3.1.

\section{References}

[BaMu92] R. Balasubramanian and V. Kumar Murty, Zeros of Dirichlet L-functions, Ann. Scient. Ecole Norm. Sup., 25 (1992), 567-615.

[BaVe68] M. B. Barban and P. P. Vehov, On an extremal problem, Trans. Moscow Math. Soc., 18 (1968), 91-99.

[Gr78] S. Graham, An asymptotic estimate related to Selberg's sieve, J. Number Theory, 10 (1978), 83-94.

[Gr81] S. Graham, On Linnik's constant, Acta Arith., 39 (1981), 163-179.

[Ju77] M. Jutila, On Linnik's constant, Math. Scand., 41 (1977), 45-62.

[Mo74] Y. Motohashi, On a problem in the theory of sieve methods, Kōkȳuroku RIMS, 222 (1974), 9-50 (Japanese).

\section{Kumar Murty}

Department of Mathematics

University of Toronto, 40 St. George Street

Toronto, CANADA M5S 2E4

e-mail: murty@math.toronto.edu 